\title{
Complex 3D scan trajectories for industrial cone-beam computed tomography using a hexapod
}

\author{
Lorenz Butzhammer* (i) and Tino Hausotte $\mathbb{D}$ \\ Institute of Manufacturing Metrology (FMT), Friedrich-Alexander-Universität Erlangen-Nürnberg (FAU), \\ Nägelsbachstraße 25, 91052 Erlangen, Germany \\ E-mail: Lorenz.Butzhammer@fmt.fau.de
}

Received 25 February 2021, revised 21 May 2021

Accepted for publication 7 June 2021

Published 29 June 2021

\begin{abstract}
Industrial x-ray computed tomography (CT) represents an established measurement technique in the field of nondestructive testing and dimensional metrology. However, the measurement accuracy is sometimes limited by measurement artifacts that cannot be avoided using a standard circular scan trajectory. This problem can be addressed with the aid of flexible 3D trajectories, but up to date, the application of these is mainly restricted to special CT devices using robot arms. In this paper, we present results using a hexapod as an additional positioning system in a commercial industrial CT scanner. In addition to the $360^{\circ}$ rotation, task-specific tilting of the part during the scan is possible in this way. We used and adapted geometry calibration procedures based on a multi-sphere reference object to enable reconstruction with high accuracy. Using a demonstrator test fixture with high absorbing elements, we show that severe metal and truncation artifacts can be avoided for a region-of-interest scan. Furthermore, cone-beam artifacts, which are inherent to circular scan trajectories, can be reduced significantly. Using measurement objects that can be measured well with a circular trajectory, we found that applying a 3D trajectory leads to dimensional measurement deviations in the same range or even lower than those of a circular trajectory. This suggests that the pose repeatability of the hexapod is sufficient to perform complex scan paths without general loss of accuracy. The obtained results could be relevant for end users of conventional CT scanners, as upgrading existing devices is in principle possible. The presented investigations form the basis for the application of trajectory optimization algorithms.
\end{abstract}

Keywords: computed tomography, dimensional metrology, scan trajectory, hexapod

(Some figures may appear in colour only in the online journal)

\section{Introduction}

Industrial x-ray computed tomography (CT) devices are increasingly widespread in use at manufacturing companies as

\footnotetext{
* Author to whom any correspondence should be addressed.
}

Original Content from this work may be used under the terms of the Creative Commons Attribution 4.0 licence. Any further distribution of this work must maintain attribution to the author(s) and the title of the work, journal citation and DOI. well as metrology laboratories. In most cases, cone-beam CT scanners with flat panel detectors are used [1]. In contrast to medical CT scanners, the $\mathrm{x}$-ray source and the detector are kept fixed in space during a scan, while a $360^{\circ}$ rotation of the measurement object is performed $[2,3]$. When choosing a coordinate system with origin on the rotation axis, the rotation of the part however can be described in the form of a circular trajectory of the source (in the following named 'scan trajectory').

There are two major drawbacks of a circular scan trajectory for metrological purposes. First, due to the $360^{\circ}$ rotation, unfavorable projective views (e.g. with high penetration lengths 
or high absorbing components obscuring the actual region of interest (ROI)) might not be avoidable for certain scan angles. Second, the Tuy sufficiency condition [4], which states that all planes through the measurement object must cut the scan trajectory, is not satisfied. Both problems can lead to strong measurement artifacts in the reconstructed volume. This can further lead to increased measurement deviations or even to the impossibility of evaluating certain measurands. Cone-beam artifacts due to the unsatisfied Tuy condition can be avoided by applying 3D scan trajectories with a regular shape. An example for such a regular shape is the helical trajectory, for which analytical reconstruction techniques exist [5]. However, the first problem of disadvantageous projective views cannot be avoided.

In contrast, more complex, task-specific, trajectories could lead to further improvements, but also demand flexible reconstruction techniques such as iterative methods [6], which in principle allow arbitrary scan trajectories. While in the medical field task-specific 3D trajectories have already been successfully applied using C-arm CT devices [7], there are only initial approaches to technical implementation for industrial purposes. Custom-built or prototype devices all use robot arms to either position the source and the detector or the part. In [8], an overview of possible arrangements is given.

There exists only little research dealing with experimental results for such setups. In [9], a robot arm is used to position the measurement object. Spherical markers are imaged along with the object to extract the pose for each projection. In [10, $11]$, both the X-ray source and the detector are positioned by a robot arm while the part is kept fixed. This arrangement can make it even impossible to perform a circular scan trajectory. A similar setup is shown in [12]. Alternatively, both the source and the detector can be mounted on a single robot as a C-arm device [13].

The possibility to scan components of large scale assemblies such as entire cars is the main benefit of using robot arms. Current research on robot arm CT systems addresses the characterization and improvement of the positioning accuracy $[8,14]$. As a precise knowledge of the geometry description for each single $\mathrm{x}$-ray projection is necessary for a high quality volume reconstruction with good structural resolution (according to guideline VDI/VDE 2630 part 1.3 defined as the smallest structure that can still be measured dimensionally [15]), the positioning accuracy of the robot arms can be seen as a major challenge.

While the technical realization of complex 3D scan trajectories is still in a comparatively early stage, research on how a 3D trajectory can be adapted for optimal reconstruction quality can already be found in the literature. Fischer et al [16] used simulated projections to optimize the trajectory for a small number of projections. The authors used an objective function that is based on the local data noise and transmitted spatial frequencies in the reconstructed volume. Herl et al [17] used the magnitude of attenuation and a criterion for the completeness of data in terms of the Tuy condition as optimization parameters. Criteria that have so far only been applied to conventional CT scans, such as radiograph-based [18], geometrically derived [19, 20] or histogram-based [21] quality parameters could also in principle be used for trajectory optimization.

3D trajectories could also be advantageous for small parts. The use of robot arms however might not be feasible in this case, especially for end users who already work with conventional industrial cone-beam CT scanners. Hexapod devices as high precision parallel manipulators with six degrees of freedom could be a promising alternative. Here, we present results using such a hexapod as an additional component to position the measurement object in a cone-beam CT scanner. While such a setup has already been applied for computed laminography of flat objects with high aspect ratio [22], it can also be used to perform $3 \mathrm{D}$ trajectories for CT scans. For this purpose, in addition to the $360^{\circ}$ rotation, the part is arbitrarily tilted by the hexapod during the scan. We investigated different measurement objects and 3D trajectories to compare various dimensional measurement tasks ranging from a case where no proper reconstruction is possible using a circular trajectory to a case where no improvements are expected for a 3D trajectory. An iterative reconstruction technique was used to account for arbitrarily shaped trajectories. To enable precise reconstruction, we used an off-line geometry calibration method based on a calibrated multi-sphere reference object. This method is a modification of common calibration procedures for cone-beam scanners, using the geometry parameters from the internal calibration of the CT device as prior knowledge. Qualitative as well as quantitative results from dimensional measurements are compared to results obtained with the conventional circular trajectory.

\section{Experimental setup}

Figure 1 shows the combined setup consisting of the conebeam CT device Metrotom 1500 G2 (Carl Zeiss Industrielle Messtechnik GmbH, Oberkochen, Germany) and the hexapod H-840.G2 (Physik Instrumente (PI) GmbH \& Co. KG, Karlsruhe, Germany). The CT device is equipped with an $\mathrm{x}$ ray tube with a maximum voltage of $225 \mathrm{kV}$ and a flat panel detector with $2048^{2}$ pixels of size $(0.2 \mathrm{~mm})^{2}$. The fixed distance between the spot of the x-ray source and the detector is roughly $1380 \mathrm{~mm}$. The hexapod is mounted on top of the rotary table of the CT device by means of an adapter plate that was supplied by the CT manufacturer. Winding of the supply cables during the $360^{\circ}$ rotation of the rotary table is supported by a cable routing and a metal tube with enclosed lead, which additionally acts as a shield to protect the encoders from secondary radiation.

The hexapod allows for motions with six degrees of freedom with the restriction of a travel range of $\pm 50 \mathrm{~mm}$ $( \pm 25 \mathrm{~mm})$ in horizontal (vertical) direction and a maximum rotational range of $\pm 15^{\circ}\left( \pm 30^{\circ}\right)$ around the horizontal (vertical) axis. This maximum angular range can only be reached at the spatial zero position of the hexapod. Consequently, the limitations of the hexapod movement narrow realizable points for the scan trajectory. In our work, we kept the position of the hexapod stage constant during a CT scan and restricted 


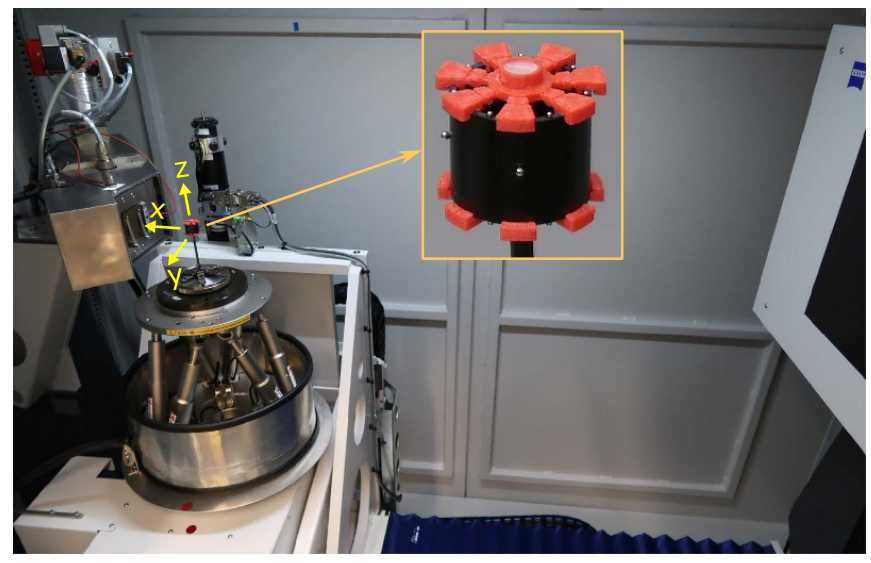

Figure 1. Hexapod as manipulator system on top of the rotary table of the cone-beam CT device between X-ray tube (left) and detector (right). On top of the hexapod, the multi-sphere reference object that was used for trajectory calibration (kindly provided by METAS) is visible. The drawn in axes are the ones of the world coordinate system $\mathcal{W}$ used later.

rotations to a tilt around the horizontal axis perpendicular to the source-detector direction ( $y$-axis in figure 1). However, in principle any kind of rotation and spatial position could be combined using the calibration method described later. The hexapod was controlled using a programmable interface of the CT device. In this way, both the CT device as well as the hexapod can be controlled using commands written in C\#. The acquired $\mathrm{x}$-ray projection images were preprocessed with a tool provided by the CT supplier to correct the image distortion according to [23].

It is worth to compare the maximum rotational range of the hexapod with the cone angle of the CT device, which is roughly $17^{\circ}$. To satisfy the Tuy condition and avoid cone-beam artifacts even for horizontal surfaces which are at initial state imaged at the outermost (top or bottom) line of the detector, the measurement object has to be tilted at least by half of the cone-angle. For the used settings, this condition could always be satisfied.

The measurement object has to be mounted keeping a certain distance from the hexapod stage to avoid that the part is obscured by parts of the tilted stage in the x-ray image. It is therefore convenient to set a Pivot point (center point of the rotational system). This point can be set roughly to the center of the measurement object. In this way, the object can be tilted without changing the position, respectively magnification. However, with increasing height of the Pivot point, the range of the rotation angles that can be applied is narrowing. For the highest distance from the stage used in our investigations $(144 \mathrm{~mm})$, the applicable tilt angle range was decreased from $\pm 15^{\circ}$ to $\pm 12^{\circ}$.

We used three different measurement objects for our examinations. These were chosen to cover a wide range of different challenges and measurement tasks. The objects are shown in the upper row of figure 2 in their mounted state. The used trajectory paths and exemplary x-ray images can be found in the middle and bottom row of this figure. In each case, a 3D trajectory as well as a circular trajectory were performed for direct comparison.

To test and demonstrate possible advantages of a complex 3D scan trajectory, the first part (plastic brick 4211210/3003, Lego System A/S) was placed inside a demonstrator test fixture consisting of an aluminum ring and several steel pins (see top of figure 2(a)). This fixture was constructed in a way that in case of a circular trajectory, the measurement object is obscured by metal components in the x-ray image at certain scan angles (see left x-ray image in the bottom row of figure 2(a)). There is no conventional circular trajectory where this can be avoided. A potential solution for this problem could be to omit scan angles that lead to an overlapping of the projected steel pins with the part. Hence, we additionally reconstructed the object using only task-specifically selected x-ray images of the original projection stack, similar as in former investigations [24]. All x-ray images with obstructed view were therefore sorted out. As a consequence, the number of used x-ray images decreased to roughly one third of the number for the full scan.

On the other hand, unobstructed views for the full scan can be achieved using a 3D trajectory. We used the scan trajectory illustrated in the middle row of figure 2(a) for that purpose. This scan trajectory is realized by section-wise tilting of the part with an alternating angle of $\pm 15^{\circ}$. The transitions between these two tilt angles are carried out as a linear ramp extending over a scan angle range of $9.5^{\circ}$ respectively. In these ramp regions, $\mathrm{x}$-ray images are acquired with a constant scan angle increment, just as for the plateau regions of the maximum/minimum tilt angle. For the used magnification, the metal components were not fully covered by the detector for the used trajectories. This means that e.g. the steel pins moved into and out of the field of view. Thus, this setup can be regarded as ROI scan, where truncation artifacts are expected. To be able to compare the results with scans without obscured views, the steel pins can be removed without displacing the measurement object. Reconstructed volume data for the different scan trajectories will be compared using cross sections and 3D renderings of the part after surface determination.

The second object, a steel ball (grade G10 according to DIN 5401 [25]) with a diameter of $8 \mathrm{~mm}$, was chosen to account for possible improvements in terms of cone-beam artifacts. Spheres are objects that cannot be oriented in such a way that cone-beam artifacts can be avoided for a circular scan trajectory. The projection of the ball was by purpose not perfectly centered in the detector image to get more pronounced cone-beam artifacts at the lower pole. The magnification was not increased further, because the projection of the multisphere reference object used for calibration (see next section) would have exceeded the detector area. The tilt angle was varied sinusoidally between $\pm 8^{\circ}$. The ball's diameter as well as the sphericity (minimum difference between the radii of two concentric spheres that enclose all fit points) were defined as measurement tasks. Besides quantitative results for these measurands, the spatial distribution of fit point deviations as well as cross sections through the reconstructed volume will be compared to assess the influence of the scan trajectory on the appearance of cone-beam artifacts. To investigate possible 

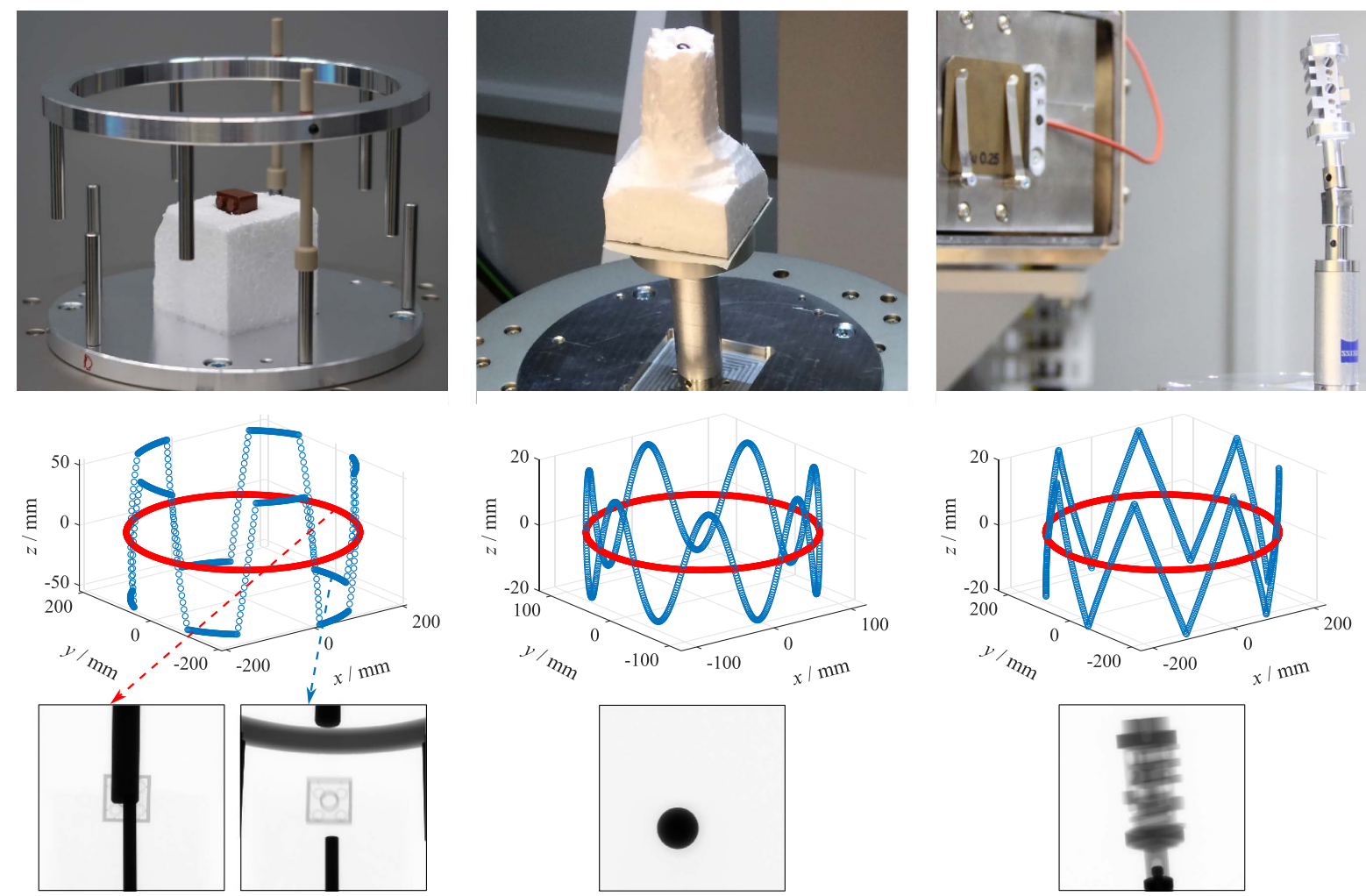

(a)

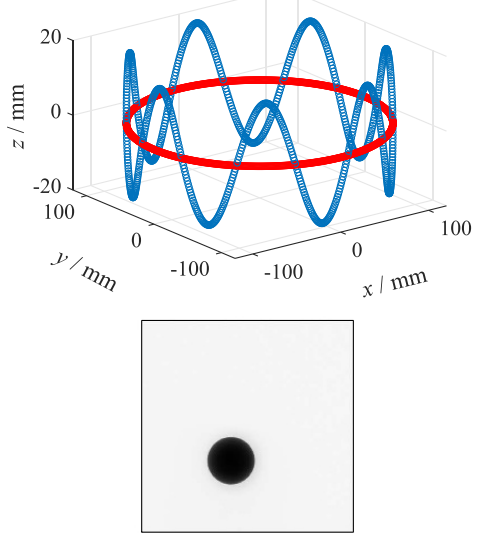

(b)

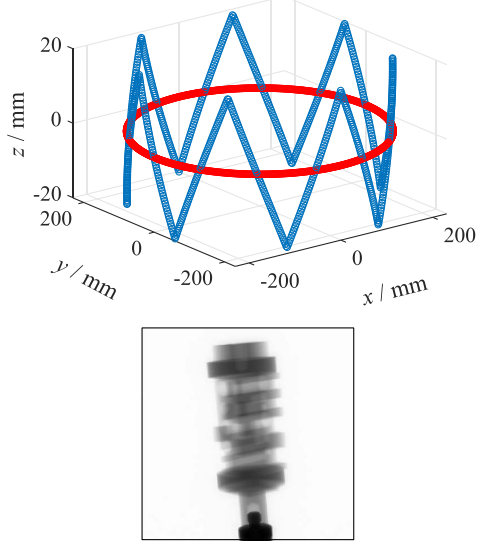

(c)

Figure 2. Overview of the investigated measurement objects (upper row), scan trajectories (middle row) and exemplary x-ray images (lower row). (a) Plastic brick in demonstrator test fixture with high absorbing (removable) steel pins. (b) Steel ball (8 mm). (c) Aluminium multi-feature specimen.

influences on the dimensional measurement results, not only the applied scan trajectory was varied for the steel ball, but also the number of iterations for the algebraic iterative reconstruction. Moreover, the trajectory calibration procedure outlined in the next section was also performed for the circular scan trajectory, for which otherwise an ideal circle with constant scan angle increment between consecutive projections is assumed. To further test the robustness of the trajectory calibration procedure, calibration scans were performed with and without binning of $2 \times 2$ pixels of the detector as well as chronologically before and after the measurements of the ball. The measurement object was then reconstructed multiple times using the different calibration scans. Differences in dimensional measurement results caused by the trajectory calibration procedure can be extracted in this way.

Finally, a multi-feature specimen made from aluminum (EN AW-6082) was chosen as measurement object (see figure 2(c)). We used several unidirectional and bidirectional length features from 2 to $10 \mathrm{~mm}$ for our investigations. These measurands are based on least squares fitted planes. The distances were measured along the respective axis of the part. The location and nomenclature for the measurands can be found in figure 3. The part was fixed with an initial orientation that was found to be optimal in terms of geometrically derived quality parameters for a circular trajectory in former investigations [20]. For this orientation, no obvious measurement artifacts are visible for the circular trajectory. Therefore, it is not expected that a 3D scan trajectory leads to an improved imaging quality for the reconstructed volume. As no significant differences between the different scan trajectories in terms of imaging artifacts are expected, this setup can be seen as test case to get a hint on how much inaccuracies from the hexapod axes influence measurement deviations.

For both the steel ball and the multi-feature specimen, ten measurement repetitions were done. To exclude systematic effects from changing conditions, the measurement repetitions for the two different scan trajectories were performed in alternating order.

Reference values for the diameter of the steel ball as well as for all measurands of the multi-feature specimen were obtained using the tactile coordinate measurement machine (CMM) UPMC 1200 CARAT S-ACC (Carl Zeiss Industrielle Messtechnik GmbH, Oberkochen, Germany). The expanded measurement uncertainties (coverage factor $k=2$ ) for the reference values were determined using Monte Carlo simulations (virtual CMM according to guideline VDI/VDE 2617 part 7 [26]). In case of the sphericity of the steel ball, no reference measurement was done. Measurements with CT showed form deviations that are always more than ten times larger than the manufacturing tolerance for the used grade $(0.25 \mu \mathrm{m})$. Therefore, the measurement values for the sphericity were directly used for comparisons. 

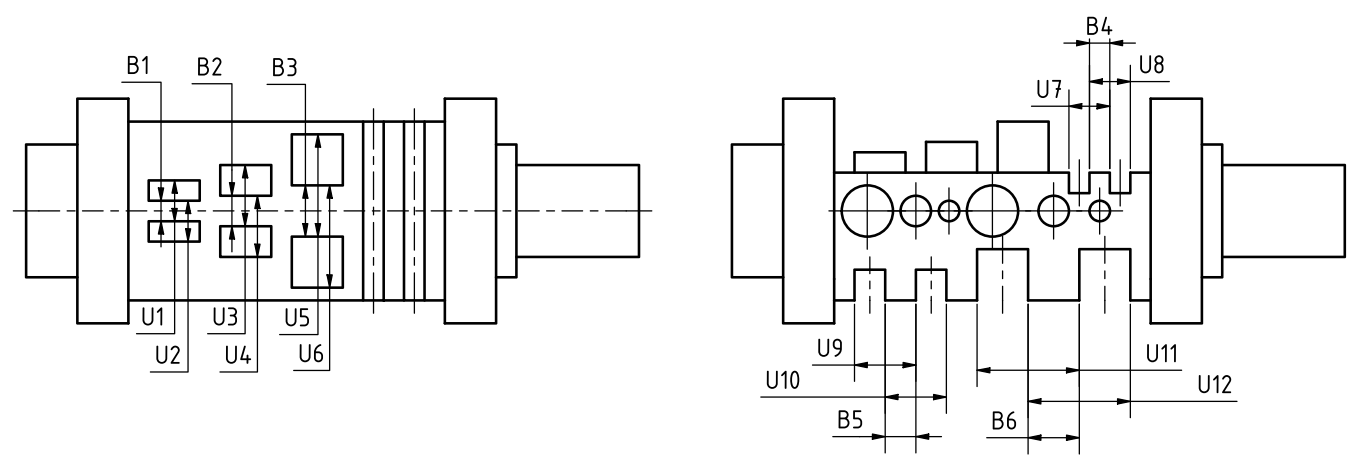

Figure 3. Evaluated unidirectional (U) and bidirectional (B) length features for the multi-feature specimen.

The used scan parameters for all scans are summarized in table 1 . All scans were reconstructed using the application programming interface of the software CERA 6 (Siemens Healthcare $\mathrm{GmbH}$, Erlangen, Germany). The reconstructions were done on a computer with an AMD Ryzen 7 2700X processor, 32 GB RAM and the graphics processing unit NVIDIA GeForce GTX 1070 (8 GB memory). Both algebraic iterative reconstruction as well as Feldkamp-Davis-Kress ( FDK) reconstruction [27] were used in case of circular scan trajectories. For iterative reconstruction, we used the algebraic reconstruction pipeline of CERA with algorithm setting 'basic', which is a 'typical adoption of the standard simultaneous algebraic reconstruction technique (SART) known in literature' [28]. The theoretical background for this reconstruction technique can be found in [29]. In the following, the abbreviation SART is used. Due to the complex form of the 3D trajectories, only iterative reconstruction could be used for this case. Projection matrices were used to feed the projection geometry to the reconstruction algorithm (see section 3 ). The reconstruction of the volume was restricted to the actual ROI around the measurement objects. Due to the limited memory size of the used graphics card, which also limits the maximum number of voxels for the volume to be iteratively reconstructed, two of the three objects were scanned using $2 \times 2$ pixel binning of the detector. SART reconstructions were performed using a relaxation factor of 0.5 (plastic brick) or 0.8 (steel ball, aluminum part). The scan times and reconstruction times for the different measurement objects and scan trajectories are listed in table 2. Dimensional measurements were done using the software VGStudio Max 3.4 (Volume Graphics GmbH, Heidelberg, Germany). Local adaptive thresholds were thereby used for automatic surface determination.

\section{Trajectory calibration}

The major problem for reconstructing the measurement volume from a 3D trajectory is that the position and orientation (pose) of the additional manipulator system (in our case the hexapod) with regard to the CT coordinate system is not known in advance. Thus, it is also not known how the measurement object moves in relation to the CT coordinate system if a certain transformation is set for the hexapod stage. However, exactly this information is needed for accurate reconstruction.
Table 1. Used scan parameters. The nomenclature for the measurement objects is according to figure 2 .

\begin{tabular}{llll}
\hline Measurement object & $\mathrm{a}$ & $\mathrm{b}$ & $\mathrm{c}$ \\
\hline Number of projections & 720 & 900 & 900 \\
X-ray tube voltage $(\mathrm{kV})$ & 100 & 210 & 180 \\
X-ray tube current $(\mu \mathrm{A})$ & 350 & 85 & 370 \\
Prefilter thickness $(\mathrm{Cu})(\mathrm{mm})$ & - & 0.25 & 0.25 \\
Integration time $(\mathrm{s})$ & 0.7 & 2.0 & 1.0 \\
Gain & 8 & 8 & 8 \\
Pixel binning & $2 \times 2$ & $1 \times 1$ & $2 \times 2$ \\
Magnification & 6.6 & 11.4 & 6.0 \\
Voxel side length $(\mu \mathrm{m})$ & 61.1 & 17.5 & 66.9 \\
Maximum tilt angle $\left({ }^{\circ}\right)$ & 15 & 8 & 5 \\
\hline
\end{tabular}

There are different possibilities to solve this problem. If the repeatability of the manipulator system is not sufficient, markers could be imaged simultaneously along with the measurement object [9]. In our case, we assume that the pose repeatability of the hexapod is good enough that off-line calibration procedures (calibrating the scan geometry before or after the actual measurement scan) can be applied. The specifications of the supplier (max. $\pm 0.5 \mu \mathrm{m}$ and $\pm 12 \mu \mathrm{rad}$ ) and results in the literature using a similar hexapod for Computed Laminography [30] suggest that this assumption is valid.

In the case of off-line calibration procedures, again different methods can in principle be applied. Blumensath et al [30] proposed a method for determining the position and orientation of the manipulator system by means of a multi-step optimization approach using the projections of spherical markers with unknown positions in $3 \mathrm{D}$.

Another possibility, which has finally been applied for our investigations, is to use a calibrated multi-sphere reference object. As the positions of the spheres are known in the coordinate system of the multi-sphere object, the parameters of the projective transformation for a specific point of the scan trajectory can be determined from a single projection [31]. A detailed description of how this can be implemented to extract the geometry parameters for a cone-beam setup can be found in the publication of Li et al [32]. Applying this procedure on each single point of the trajectory makes it possible to extract the experimentally realized source trajectory (in relation to the multi-sphere object) for the full scan without knowledge of the 
Table 2. Scan times and reconstruction times for the different measurement objects and scan trajectories. The reconstruction time for the $3 \mathrm{D}$ scan trajectory is given for the numbers of iterations (It.) that were used in our investigations.

\begin{tabular}{|c|c|c|c|c|}
\hline \multirow[b]{2}{*}{ Measurement object } & \multicolumn{2}{|c|}{ Scan time (min) } & \multicolumn{2}{|c|}{ Reconstruction time (s) } \\
\hline & Circ. traj. & $3 \mathrm{D}$ traj. & Circ. traj. (FDK) & 3D traj. (SART) \\
\hline (a) Plastic brick & 34 & 49 & 22 & 42 (1 It.) \\
\hline (b) Steel ball & 70 & 95 & 119 & $\begin{array}{l}137 \text { (1 It.) } \\
626 \text { (5 It.) }\end{array}$ \\
\hline (c) Multi-feature spec. & 48 & 63 & 40 & 223 (5 It.) \\
\hline
\end{tabular}

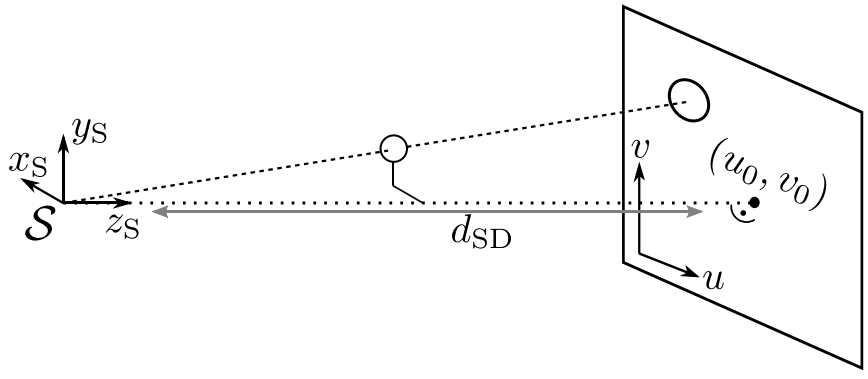

Figure 4. General schematic for a cone-beam projection.

position of the hexapod coordinate system. This means that at least two scans are necessary to reconstruct a measurement object from a 3D scan trajectory. First, the multi-sphere reference object has to be scanned to extract the scan geometry. Second, the measurement object itself has to be scanned using the identical trajectory. As we also applied the reconstruction in an off-line manner, the order for the calibration scan and the measurement scan can also be changed.

In the following, we show how we implemented and adapted the methods described in [32]. The mathematical framework is based on the description given in [31]. The multisphere reference object we used consists of 17 metal spheres on a carbon fiber tube and was kindly provided by the Swiss Federal Institute of Metrology METAS. The provided calibration data for the sphere positions was obtained with a microCMM [33]. Details about the multi-sphere object can be found in [34].

Figure 4 illustrates the general geometric setup for a conebeam projection onto a flat-panel detector.

Using homogeneous vectors, the projection of a point $\boldsymbol{X}_{\mathrm{S}}=$ $\left(x_{\mathrm{S}}, y_{\mathrm{S}}, z_{\mathrm{S}}\right)^{T}$ in the 3D source coordinate system $\mathcal{S}$ to a point $(u, v)^{T}$ in the 2D detector coordinate system can be described by multiplication with a $3 \times 4$ projection matrix $P_{\mathrm{S}}$ :

$$
\begin{aligned}
\left(\begin{array}{c}
u w \\
v w \\
w
\end{array}\right) & =P_{\mathrm{S}} \boldsymbol{X}_{\mathrm{S}}=K[I \mid \mathbf{0}] \boldsymbol{X}_{\mathrm{S}} \\
& =\left[\begin{array}{cccc}
-\frac{d_{\mathrm{SD}}}{p_{u}} & 0 & u_{0} & 0 \\
0 & \frac{d_{\mathrm{SD}}}{p_{v}} & v_{0} & 0 \\
0 & 0 & 1 & 0
\end{array}\right]\left(\begin{array}{c}
x_{\mathrm{S}} \\
y_{\mathrm{S}} \\
z_{\mathrm{S}} \\
1
\end{array}\right) .
\end{aligned}
$$

The parameter $w$ can be seen as scaling factor and is only needed as intermediate step to calculate $u$ and $v$. The left

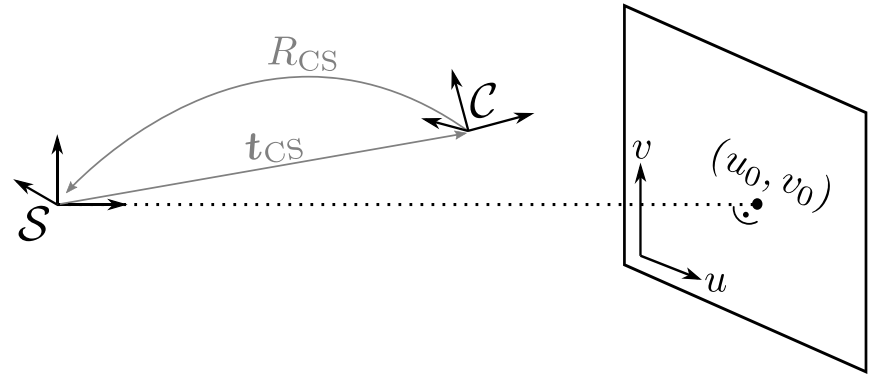

Figure 5. Coordinate system $\mathcal{C}$ of the calibrated multi-sphere reference object, connected to the source coordinate system via rotation $R_{\mathrm{CS}}$ and shift $t_{\mathrm{CS}}$.

$3 \times 3$ submatrix of $P_{\mathrm{S}}$ contains the intrinsic geometric parameters and is named $K . I$ is the $3 \times 3$ identity matrix. $\left(u_{0}, v_{0}\right)^{T}$ represents the piercing point (point where the central ray from the source hits the detector with right angle), $d_{\mathrm{SD}}$ is the distance from source to detector and $\left(p_{u}, p_{v}\right)$ are the pixel side lengths in the two directions. We assume that the pixel elements of the detector are perfectly rectangular (no skew). Note that in contrast to [31], the first entry of the projection matrix has a negative sign because the unit vector $\boldsymbol{e}_{u}$ points in the opposite direction of $\boldsymbol{e}_{x_{\mathrm{S}}}$. If we further assume quadratic pixels and set $p_{u}=p_{v}$ to a constant value, then there are three degrees of freedom left for $K$.

In general, the task is to determine the unknown projection matrix from the correspondence between known points in $3 \mathrm{D}$ and their measured projections in $2 \mathrm{D}$. In case of a multisphere object, the coordinates of the centers of the spheres are used. As these coordinates are not known in $\mathcal{S}$, but only in the coordinate system $\mathcal{C}$ of the multi-sphere object (see figure 5), the transformation between the two coordinate systems has also to be determined.

The mapping between points in $\mathcal{C}$ and their projections can be described as:

$$
\left(\begin{array}{c}
u w \\
v w \\
w
\end{array}\right)=P_{\mathrm{C}} \boldsymbol{X}_{\mathrm{C}}=K\left[R_{\mathrm{CS}} \mid \boldsymbol{t}_{\mathrm{CS}}\right]\left(\begin{array}{c}
x_{\mathrm{C}} \\
y_{\mathrm{C}} \\
z_{\mathrm{C}} \\
1
\end{array}\right)
$$

with a $3 \times 3$ rotation matrix $R_{\mathrm{CS}}$ describing the orientation and a vector $\boldsymbol{t}_{\mathrm{CS}}$ representing the shift between the two coordinate systems. Consequently, six degrees of freedom (three for the position and three for the orientation) are added to the projection matrix. 


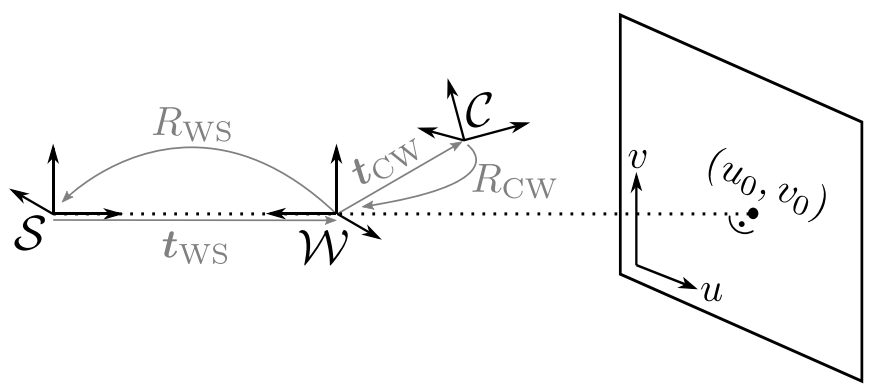

Figure 6. Used world coordinate system $\mathcal{W}$ with origin at intersection of central ray and assumed rotation axis of the rotary table.

The projection matrix $P_{\mathrm{C}}$ or rather the nine independent geometric parameters could now be determined by optimization, minimizing the geometric error:

$$
\min _{P_{\mathrm{C}}} \sum_{i} \mathrm{~d}\left(\boldsymbol{x}_{i}, P_{\mathrm{C}} \boldsymbol{X}_{\mathrm{C}, i}\right)^{2}
$$

Here, $\boldsymbol{X}_{\mathrm{C}, i}$ is the calibrated position of sphere $i$ in $3 \mathrm{D}, \boldsymbol{x}_{i}$ is the corresponding measured position on the detector and the operator $\mathrm{d}$ indicates the Euclidean distance between the measured and the projected point on the detector.

In preliminary tests we found that calibrating the full system with all degrees of freedom led to comparatively low accuracy. Therefore, we kept the intrinsic parameters fixed and relied on the internal calibration results of the CT device, which are expected to be highly accurate. The intrinsic parameters from this internal calibration are obtained with a special multi-sphere object with a high number of spheres. This object is additionally imaged at several different poses. However, we could not use this object for our own calibration procedure for $3 \mathrm{D}$ trajectories, as the arrangement of the spheres exclude the application of certain viewing angles.

Furthermore, it is convenient to not use the coordinate system of the multi-sphere reference object for reconstruction, but the world coordinate system $\mathcal{W}$ (see figure 6). This coordinate system is also automatically used for the reconstruction of circular scans. The origin of $\mathcal{W}$ is located at the intersection of the central ray (defined by the connection between source and piercing point) with the assumed rotation axis of the rotary table. The orientation of the axes was chosen to match the orientation that is inherently used by the reconstruction software. The orientation is indicated in figure 1 .

The introduction of the additional coordinate system leads to an intermediate step in the transformation from $\mathcal{C}$ to $\mathcal{S}$ and (2) can be adapted to:

$$
\begin{aligned}
\left(\begin{array}{c}
u w \\
v w \\
w
\end{array}\right) & =P_{\mathrm{C}} \boldsymbol{X}_{\mathrm{C}} \\
& =K\left[R_{\mathrm{WS}} \mid \boldsymbol{t}_{\mathrm{WS}}\right]\left[\begin{array}{cc}
R_{\mathrm{CW}} & \boldsymbol{t}_{\mathrm{CW}} \\
\mathbf{0}^{T} & 1
\end{array}\right]\left(\begin{array}{c}
x_{\mathrm{C}} \\
y_{\mathrm{C}} \\
z_{\mathrm{C}} \\
1
\end{array}\right) .
\end{aligned}
$$

The meaning of $R_{\mathrm{WS}}, \boldsymbol{t}_{\mathrm{WS}}, R_{\mathrm{CW}}$ and $\boldsymbol{t}_{\mathrm{CW}}$ can be derived from figure $6 . R_{\mathrm{WS}}$ and $\boldsymbol{t}_{\mathrm{WS}}$ are both treated as known. The shift is set to $t_{\mathrm{WS}}=\left(0,0, d_{\mathrm{SR}}\right)^{T}$ with $d_{\mathrm{SR}}$ being the distance from the source to the rotation axis (taken from the result of the internal calibration of the $\mathrm{CT}$ device). Again, only one rotation matrix $\left(R_{\mathrm{CW}}\right)$ and one shift vector $\left(t_{\mathrm{CW}}\right)$ have to be found by optimization for each projection. To account for the projection number, an index $j$ will be added in the following.

After calibration, the found projection matrices could be directly fed to the reconstruction software. However, this would result in volume data that is aligned to and centered in the coordinate system $\mathcal{C}$. To achieve that the measurement object for a 3D trajectory has exactly the same position and orientation in the voxel data set like for a circular trajectory, projection matrices describing the mapping from points in $\mathcal{W}$ to points on the detector are needed.

As for the $j$ th projection:

$$
\boldsymbol{X}_{\mathrm{W}, j}=\left[\begin{array}{cc}
R_{\mathrm{CW}, j} & \boldsymbol{t}_{\mathrm{CW}, j} \\
\mathbf{0}^{T} & 1
\end{array}\right] \boldsymbol{X}_{\mathrm{C}},
$$

this can be rearranged to:

$$
\boldsymbol{X}_{\mathrm{C}}=\left[\begin{array}{cc}
R_{\mathrm{CW}, j}^{-1} & -R_{\mathrm{CW}, j}^{-1} \boldsymbol{t}_{\mathrm{CW}, j} \\
\mathbf{0}^{T} & 1
\end{array}\right] \boldsymbol{X}_{\mathrm{W}, j} .
$$

In the reconstruction software, the first projection defines the position and orientation of the measurement object in the volume data. The envisaged registration can therefore be reached by inserting above equation with $j=1$ in (4):

$$
\begin{aligned}
\left(\begin{array}{c}
u_{j} w_{j} \\
v_{j} w_{j} \\
w_{j}
\end{array}\right)= & P_{\mathrm{W}, j} \boldsymbol{X}_{\mathrm{W}, j} \\
= & K\left[R_{\mathrm{WS}} \mid \boldsymbol{t}_{\mathrm{WS}}\right]\left[\begin{array}{cc}
R_{\mathrm{CW}, j} & \boldsymbol{t}_{\mathrm{CW}, j} \\
\mathbf{0}^{T} & 1
\end{array}\right] \\
& \times\left[\begin{array}{cc}
R_{\mathrm{CW}, 1}^{-1} & -R_{\mathrm{CW}, 1}^{-1} \boldsymbol{t}_{\mathrm{CW}, 1} \\
\mathbf{0}^{T} & 1
\end{array}\right]\left(\begin{array}{c}
x_{\mathrm{W}, j} \\
y_{\mathrm{W}, j} \\
z_{\mathrm{W}, j} \\
1
\end{array}\right) .
\end{aligned}
$$

The equation accounts for the fact that $\mathcal{C}$ and $\mathcal{W}$ do not coincide for the first projection (including a possible tilt of the hexapod). This initial discrepancy is reversed for every projection $j$ and therefore the coordinate system of the reconstructed volume is exactly the same like that for a circular trajectory. For $j=1$, it can be seen that (7) reduces to:

$$
\left(\begin{array}{c}
u_{j} w_{j} \\
v_{j} w_{j} \\
w_{j}
\end{array}\right)=K\left[R_{\mathrm{WS}} \mid \boldsymbol{t}_{\mathrm{WS}}\right]\left(\begin{array}{c}
x_{\mathrm{W}, j} \\
y_{\mathrm{W}, j} \\
z_{\mathrm{W}, j} \\
1
\end{array}\right),
$$

which is identical to (2), if $\mathcal{C}$ coincides with $\mathcal{W}$.

In summary, the projection matrices $P_{\mathrm{C}, j}$ or rather the unknown geometric parameters $\left(R_{\mathrm{CW}, j}, t_{\mathrm{CW}, j}\right)$ have to be found by optimization. Once determined, these parameters together with the fixed parameters $\left(K, R_{\mathrm{WS}}, t_{\mathrm{WS}}\right)$ can be inserted in (7) to obtain the projection matrices $P_{\mathrm{W}, j}$, which are finally fed to 
the reconstruction software to reconstruct measurement scans using the same trajectory.

For our optimization approach, we used the nonlinear leastsquares solver of the software Matlab R2020a (The MathWorks, Inc., Natick, USA) to extract $\boldsymbol{t}_{\mathrm{CW}, j}$ and the three orientation angles of a consecutive rotation describing $R_{\mathrm{CW}, j}$ out of the (at maximum) 17 point correspondences of the multisphere reference object. The geometric error (3) was used as objective function. For $\boldsymbol{X}_{\mathrm{C}}$, we directly used the center positions of the spheres from the calibration data of the multisphere object. To obtain the coordinates $(u, v)^{T}$ of the projected centers, first the contours of the projected spheres were detected. If overlap occurred, we excluded affected spheres. To detect the contours, an initial rough detection was done using a built-in function in Matlab to detect circles. After that, Canny edge detection [35] was performed locally at the relevant regions of interest.

There are two things that have to be considered to determine the projected sphere center out of the contour. First, the cone-beam projection of a sphere whose center is not on the central ray leads to an ellipsoid contour. Second, the projection of the sphere center and the midpoint of the ellipse do not coincide [36]. We considered the first point by fitting an ellipse using singular value decomposition. For this purpose we followed the method described in [32]. The second point was considered by applying a correction to the found ellipse midpoints according to [36]. To obtain the required local detector coordinate system that was used in [36], for each projected sphere the line from the known piercing point to the center of the ellipse was used to define the direction of the relevant axis, along which the correction is performed. We verified the correction method numerically for off-axis sphere projections and tilted detector geometry. However, for the conducted calibration scans, the influence of the correction on the resulting geometric error after optimization was found to be negligible compared to the variation of the error between different projections (hexapod poses). In total, no improvement in terms of the geometric error could be observed. The reason might be that in our case the ellipses were very close to a circular shape (length difference between axes in sub-pixel range). Thus, the correction is rather small and inaccuracies of the ellipse fitting can more easily lead to an imperfect correction.

In the following section, dimensional measurement results obtained with the described trajectory calibration procedure will be compared to results that are obtained assuming an ideal geometry for the circular scan trajectory. It is therefore of interest whether a scaling error remains after the internal calibration of the CT device, which provides the intrinsic parameters and the distance between source and rotation axis $\left|\boldsymbol{t}_{\mathrm{WS}}\right|$ for different set magnifications. Such a scaling error arises if there is a systematic deviation for the assumed magnification factor $m=d_{\mathrm{SD}} /\left|\boldsymbol{t}_{\mathrm{WS}}\right|$. This leads to an erroneous voxel size and consequently to erroneous length measurements.

We applied one of the methods described in [37] to determine the scaling error. This method is based on the evaluation of sphere distance errors and was presented in [37] using a ball plate. Plotting the sphere distance errors versus the calibrated lengths, a line through the origin can be fitted. The obtained slope $a$ can be seen as relative length error. In our case, we used CT scans of the multi-sphere reference object that was also used for trajectory calibration, and evaluated all 136 sphere distances that can be constructed from the 17 spheres. Fitting was done using the linear least squares method of Matlab. Following this procedure, we obtained $a=2.1 \times 10^{-4}$ for the magnification used for the measurement series of the multi-feature specimen and $a=3.2 \times 10^{-5}$ for the magnification used for the measurement series of the steel ball. This means that for the diameter of the steel ball $(8 \mathrm{~mm})$, a measurement deviation due to scaling error of $0.3 \mu \mathrm{m}$ can be expected, while for the measurands of the multi-feature specimen, the deviations can reach $2 \mu \mathrm{m}$ (for the highest evaluated distance of $10 \mathrm{~mm}$ ). However, it must be mentioned that due to high scattering of single sphere distance errors, the goodness of fit to determine $a$ was comparatively low with a coefficient of determination [38] of $R^{2}<0.1$ for all data sets.

If we apply the method to determine the scaling error also on the realized 3D scan trajectories, the result is $a=$ $-4.9 \times 10^{-5}$ (multi-feature specimen) and $a=-1.4 \times 10^{-5}$ (steel ball). For the following results, scaling errors have not been compensated but will be considered for the discussion of dimensional measurement deviations.

\section{Results and discussion}

Qualitative results for the plastic brick are demonstrated in figure 7 in the form of vertical cross sections and 3D renderings of the part after surface determination.

The left column of figure 7 shows results if the metal frame with the steel pins is dismounted. While for the first two rows all available projections for the circular scan trajectory were used to reconstruct the object with the two different reconstruction algorithms, for the third row only those scan angles were used that showed no obscuring of the part by steel components for the measurement with metal frame. In the last row, the results with 3D scan trajectory can be seen. For the case without metal frame, there are only minor differences for the imaging quality between the reconstructions, except for the circular scan with task-specific scan angles, where sampling artifacts due to the low number of projections and limited angular range appear. Having a closer look on the results for the circular scan trajectory, cone-beam artifacts are visible. They appear as streak artifacts arising from the horizontal edges of the part at the top and bottom of the cross section and are most pronounced for the FDK reconstruction. In case of the 3D scan trajectory, cone-beam artifacts have vanished, but the background appears less homogeneous in comparison to the full circular scan.

If the part is scanned with the metal frame, the reconstructed volume is strongly affected by artifacts caused by the steel pins in case of the conventional circular trajectory. The artifacts are a consequence of the high absorption of the pins and the fact that the pins are not completely imaged for all scan angles. This leads to inconsistencies in the reconstruction data that further implicate truncation artifacts. For both FDK and SART reconstruction of the circular scan, the brick is only 


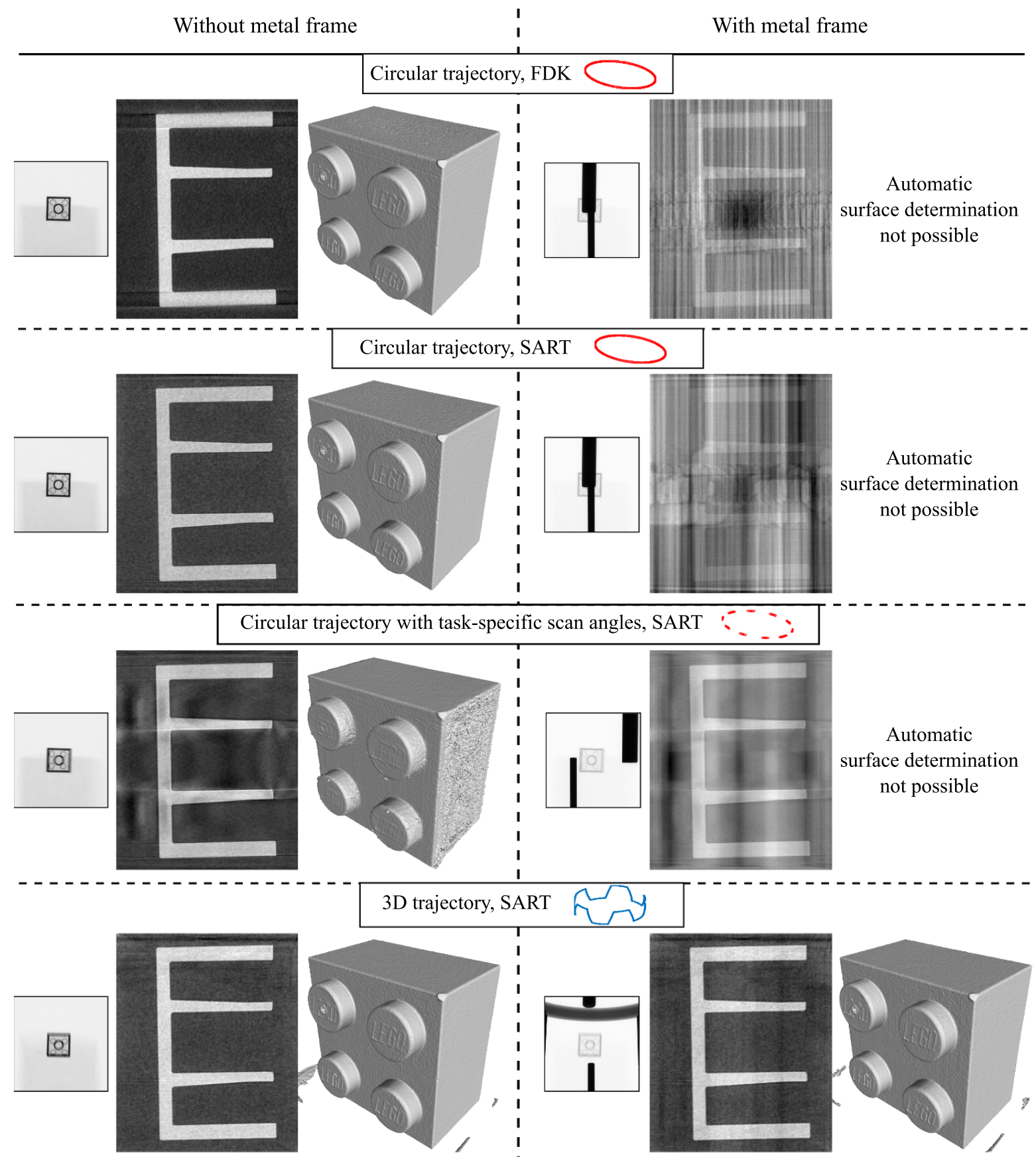

Figure 7. Qualitative results for the plastic brick using different trajectories (see figure 2(a)) and reconstruction algorithms. In case of the left column, the metal frame of the demonstrator test fixture was dismounted. The first flat-field corrected $\mathrm{x}$-ray image of each scan is imaged next to a vertical cross section through the reconstructed volume and a 3D representation of the part. The gray values of each cross section are linearly mapped between the minimum value (black) and the maximum value (white).

weakly visible. The artifacts make it impossible to extract the surface of the part.

If task-specific scan angles are used for the circular scan trajectory, artifacts arising from the steel pins are widely reduced at the region of the measurement object but the overall image quality is too low to enable a proper detection of the surface of the part.

If the 3D scan trajectory is applied, the part is kept in 'clear view' for the full rotation with a sufficient distance to the projected steel components. This leads to a reconstruction quality that is comparable to the case without metal frame. It is noteworthy that strong artifacts can also appear in case of the 3D scan trajectory, but these artifacts are located outside the ROI and, therefore, do not affect the part.

The presented results for the plastic brick inside the demonstrator test fixture can be regarded as proof of principle and as best possible improvement that can be reached with a 3D scan trajectory. Of course, this example is an artificially created task that might not exist in such a form in reality. The metal frame has been designed specifically to illustrate how the proposed 

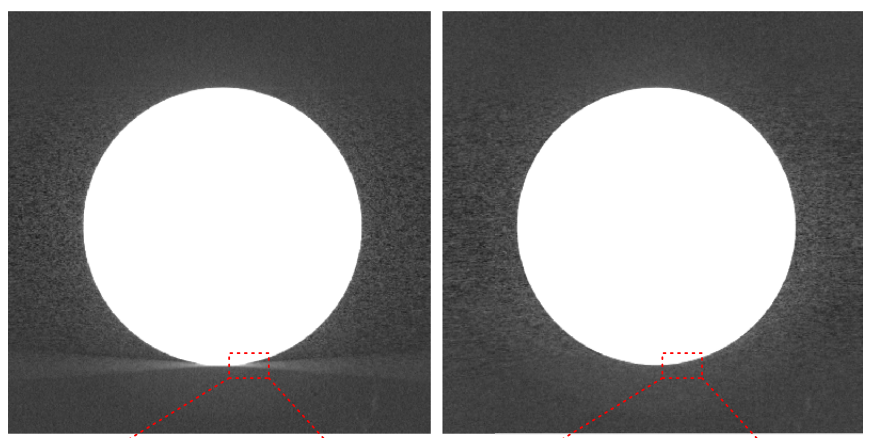

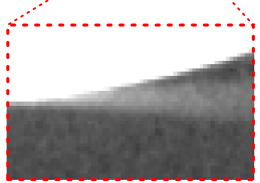

(a)

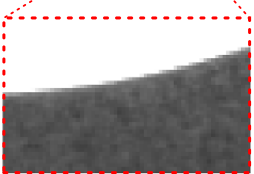

(b)
Figure 8. Vertical cross sections for the measured steel ball. (a) Circular scan trajectory with FDK reconstruction. (b) 3D scan trajectory (see figure 2(b)). For better visualization, the gray values of the cross sections are linearly mapped between the minimum value (black) and roughly $35 \%$ of the maximum (white).

method can be used to omit less favorable projections. The design of the object is not intended to be representative of real engineering components. Nevertheless, for specific cases, the used method could be an alternative to ROI CT [39]. Additional studies will be required to validate the method for real ROI scan scenarios.

To elucidate the effect of 3D trajectories on suppressing cone-beam artifacts, the steel ball was scanned at an off-center position. This position was chosen in a way that the upper pole was located close to the horizontal plane, which contains the central ray. In this way, negligible cone-beam artifacts are expected for the upper pole, while comparatively strong artifacts are expected for the lower pole in case of a circular trajectory. In the cross section of figure 8(a), which shows results for the circular trajectory, it can be seen that this is indeed the case. Streaks arising from the lower pole are clearly visible.

To extract the effects of these artifacts on dimensional measurements, the fit point deviations with regard to the fitted ideal sphere were additionally evaluated and visualized in figure 9. Figure 9(a) shows that the cone-beam artifacts lead to positive point deviations of more than $6 \mu \mathrm{m}$ at the region around the pole.

If the 3D scan trajectory with sinusoidally varying tilt angle is applied, the cone-beam artifacts as well as the high fit point deviations at the pole are completely vanishing, compare figures 8(b) and 9(b). As the maximum tilt angle of $8^{\circ}$ is greater than half of the cone angle of the CT device $\left(7.5^{\circ}\right)$, surface normals that are initially located inside the cone of missing frequencies [40] are completely rotated out of this region.

On the other hand, it is observable that the absolute values of the fit point deviations at areas outside the pole region increase. This could be due to the fact that for the used reconstruction software and chosen settings, SART reconstructions showed a lower contrast-to-noise ratio (CNR) in comparison to FDK reconstructions. The noise increases with higher number of applied iterations for the SART reconstruction, but even for a single iteration, which was applied in case of figure 9(b), the CNR is lower than the one for FDK reconstruction. Other possible reasons, which cannot be excluded purely from this data set, could be inaccuracies arising from the limited repeatability of the hexapod or from the calibration procedure. For certain projections of the calibration scan, overlapping of up to six spheres occurred. As these spheres could not be used for the optimization procedure of the calibration, higher uncertainties for the found geometry parameters of these affected projections are assumed. As such errors propagate through the backprojection process into the final volume, this could consequently yield higher deviations for the surface fit points.

To shed light on the mentioned possible influences, dimensional measurements were evaluated for the repeated scans of the steel ball. Apart from the scan trajectory, the number of iterations for the algebraic iterative reconstruction was varied. Additionally, a calibration scan was applied as well for the circular scan trajectory. Thus, the scans with circular trajectory can be iteratively reconstructed in two different ways. On the one hand, the reconstruction can be done in the conventional way that is also used for the FDK reconstruction. For this conventional way, it is assumed that the scan trajectory is represented by an ideal geometry, i.e. circle. The radius of this circle is taken from the internal calibration results of the CT device. On the other hand, projection matrices from the calibration scan can be directly applied to describe the geometry of the trajectory projection-wise, exactly as done for the 3D scan trajectory.

Figure 10 shows the results that were obtained using the different reconstruction algorithms and the different methods to describe the geometry of the scan trajectories (assuming ideal geometry or using projection matrices). In figure 10(a), the measurement deviations for the diameter are plotted. The expanded measurement uncertainty for the CMM reference measurement (which defines zero deviation in the diagram) was $U=0.6 \mu \mathrm{m}$ with a coverage factor of $k=2$. Therefore, the diameter values obtained with CT are clearly too large for all cases. The high deviations cannot be explained by a scaling error, as the scaling error for the used magnification was determined to be below $0.3 \mu \mathrm{m}$ for a length of $8 \mathrm{~mm}$ (see considerations about scaling errors in section 3). Comparing the results for the case where an ideal geometry is assumed, it can be observed that SART leads to reduced deviations but a slightly increased range. This could be caused by the different resulting imaging characteristics of the reconstruction algorithms. While in terms of CNR, the FDK reconstruction outperforms the SART reconstruction, the latter shows higher sharpness of material edges, which might result in a more accurate diameter determination. Increasing the number of iterations enhances the differences, as the noise increases, but simultaneously also the sharpness. This is supported by the results for the sphericity (see figure 10(b)). Here, the sphericity increases with higher number of iterations because of the increasing noise, which directly affects the obtained surface roughness of the ball. 

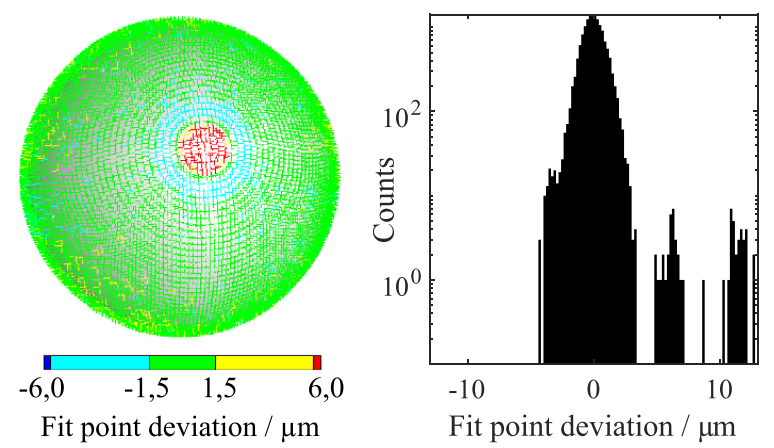

(a)
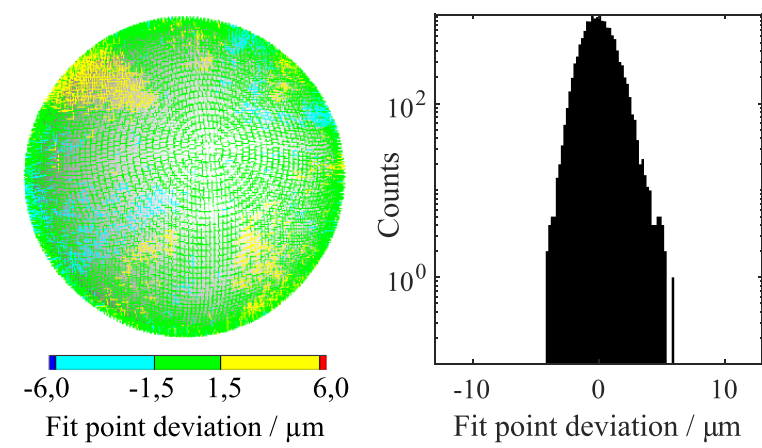

(b)

Figure 9. Spatial distribution and histogram of the fit points deviations with respect to the fitted ideal sphere for the measured steel ball. (a) Circular scan trajectory with FDK reconstruction. (b) 3D scan trajectory (see figure 2(b)). Note the grouped color scale. Fit points with deviations greater than $6 \mu \mathrm{m}$ are homogeneously colored red.

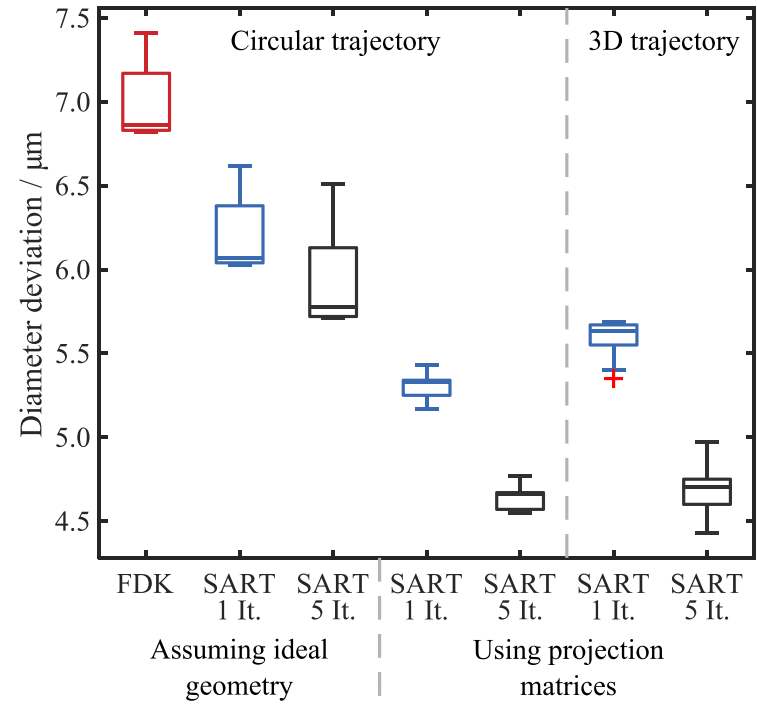

(a)

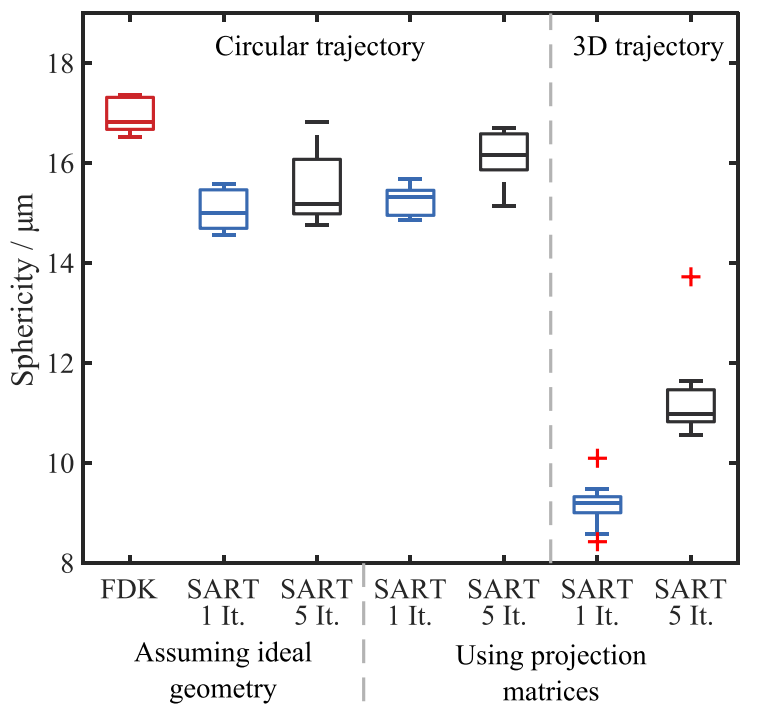

(b)

Figure 10. Measurement results for the diameter and sphericity of the steel ball for different scan trajectories and reconstruction settings $(n=10)$. The number of iterations for the iterative algebraic reconstruction was set to 1 or 5 . The scans with circular trajectory can be reconstructed assuming an ideal circle or using projection matrices found by the same calibration method that was necessarily applied to the $3 \mathrm{D}$ trajectory.

If trajectory calibration, i.e. projection matrices, are used for the circular scan trajectory, the diameter deviations are significantly reduced, while the sphericity is only slightly affected. It is assumed that the sphericity is dominated by the fit points that are affected by cone-beam artifacts, which are not reduced by only using projection matrices. Thus, the results suggest that using projection matrices leads to a more accurate determination of the projection geometry for single projections and therefore to reduced deviations for the diameter. This leads to the conclusion that the used calibration method can compensate for geometric deviations that are implied by the assumption of an ideal circle. Such geometric deviations can arise e.g. from a tilt of the rotation axis of the rotary table. It is already known in the literature, that calibration procedures based on multi-sphere reference objects can extract such deviations [41].
In comparison to the circular scan with trajectory calibration, applying the 3D scan trajectory has only minor effects on the diameter values, but leads to significant improvements for the determination of the sphericity. The latter is caused by the prevention of cone-beam artifacts, which was already shown in figures 8 and 9.

Having a closer look on the results for the diameter deviations, it can be observed that the 3D scan trajectory leads to similar advantages like using projection matrices for the circular scan. However, the medians and the range of the measurement values are slightly increased. This could be due to positioning inaccuracies caused by the limited repeatability of the hexapod. While for the circular scan the axes of the hexapod were not moved, for the 3D scan the axes were moved between two successive projections. However, as can be seen in figure 10, such possible inaccuracies have a smaller influence than e.g. the number of iterations. 


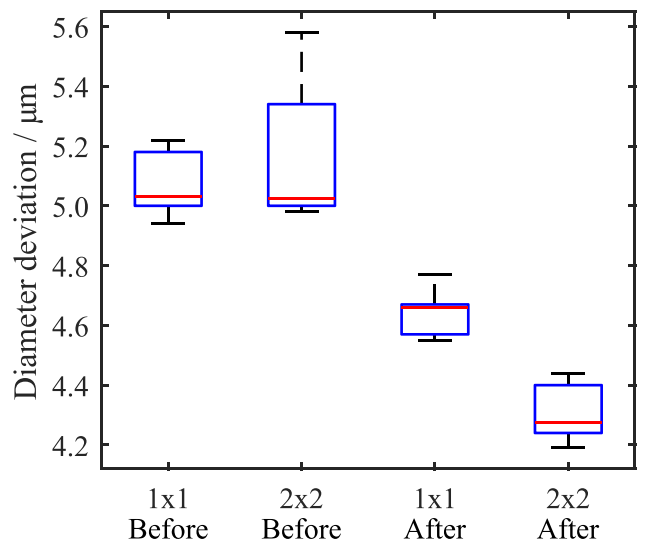

Figure 11. Influence of the used calibration scan on the diameter deviation for the steel ball (here circular scan trajectory, $n=10$ ). The calibration scan was performed before and after the measurement series, using additionally different settings for the pixel binning $(1 \times 1$ : no binning, $2 \times 2$ : binning of four pixels $)$.

To further test the influence of the trajectory calibration step, calibration scans with different setting for the detector pixel binning were performed before and after the measurement series of the steel ball. Exemplary results for the circular scan trajectory are shown in figure 11. Five iterations of the SART algorithm were used for this example. One can see that the robustness of the calibration in terms of changing conditions is limited. In comparison, using the calibration scans that were performed after the measurement series led to lower deviations. For the 3D scan trajectory similar dependencies could be observed (not shown here). The influence of the pixel binning is not clear throughout all results. Despite the influence of the conditions for the calibration on the absolute values, the general conclusions derived from figure 10 can be maintained. For all results contained in figure 10, calibration scans with $1 \times 1$ binning obtained after the measurement series were used.

To extend the basis of comparison, a greater number of measurands was analyzed using the aluminum multi-feature specimen. Furthermore, this object can be measured using a circular scan trajectory without notable artifacts. No obvious advantages of a 3D scan trajectory could be identified in advance. The choice of the applied shape of the 3D trajectory was further made without underlying physical background. Like for the steel ball, ten measurements were done for each scan trajectory. Trajectory calibration was only applied for the 3D scan trajectory because for the used magnification, a high number of overlapping spheres occurred for the circular scan. Therefore, an ideal geometry (circle) was assumed for the circular scan trajectory. While the measurement scans were performed with $2 \times 2$ pixel binning, no binning was applied for the calibration scan.

FDK as well as SART reconstruction were used for the circular scan trajectory to separate effects of the reconstruction algorithm from effects of the trajectory. In figure 12, the measurement deviations for the single measurands and different trajectories/reconstruction algorithms can be found as boxplot diagram. The measurement uncertainties of the CMM reference values are indicated by black horizontal lines for each measurand.

First, it is remarkable that the results show comparatively low absolute measurement deviations, of less than $6 \mu \mathrm{m}$ overall. In case of the unidirectional distances, two third of the median values lie inside the uncertainty range of the CMM reference value. In case of the bidirectional distances, this applies only for 5 out of 18 median values. The higher measurement deviations for bidirectional distances may originate from the higher sensitivity on inaccuracies of the surface determination. It is also visible from figure 12 that unidirectional measurands that belong to symmetric structures of the part (such as U1 and U2, see figure 3) show different results. This might be due to the fact that the part was oriented with two successive rotations around two different axes of the part. Due to this orientation, the symmetric structures were projected on different heights (detector lines). Therefore, the penetration lengths throughout the $360^{\circ}$ rotation can be different for the symmetric structures.

Looking at the results for the circular scan trajectory, it can be observed that for specific measurands, significant large differences exist, which are solely caused by the different reconstruction algorithms. Apart from some outliers of the SART data set, no clear algorithm preference can be deduced in terms of absolute deviations.

Applying the 3D scan trajectory leads to improvements for some measurands while for others the deviations increase. Similar measurands (in terms of type and axis direction, see figure 3 ) tend to show a characteristic pattern for the measurement values at different parameters. For instance, in case of measurands U7-U12, which are all unidirectional distances along the axial direction of the part, by tendency highest measurement values are obtained with circular trajectory and SART, while the lowest values are obtained with the 3D trajectory.

Considering the fact that no systematic measurement deviations can be recognized in total for the unidirectional lengths, this suggests that no significant scaling error was present for the conducted measurement series. However, the determination of the scaling error with the multi-sphere reference object (see section 3) yielded scaling errors between 0.4 and $2 \mu \mathrm{m}$ for the evaluated lengths. A compensation for the calculated errors would shift the boxes for the circular scan trajectory in figure 12 towards lower values. However, in sum, this would result in increased absolute measurement deviations. It is assumed that this is caused by an overestimation of the scaling error when evaluating the sphere distances of the multisphere object. The measured length values were therefore not corrected.

In summary, one can state that the $3 \mathrm{D}$ scan trajectory does not imply significant higher inaccuracies. As the ranges and interquartile ranges for the values obtained with the $3 \mathrm{D}$ scan trajectory are comparable to the ones obtained with circular scan trajectory, this suggests that the repeatability of the hexapod is indeed sufficient to not induce significant additional random measurement errors.

With regard to possible industrial applications of the presented methods, the impact on the measurement time is of 


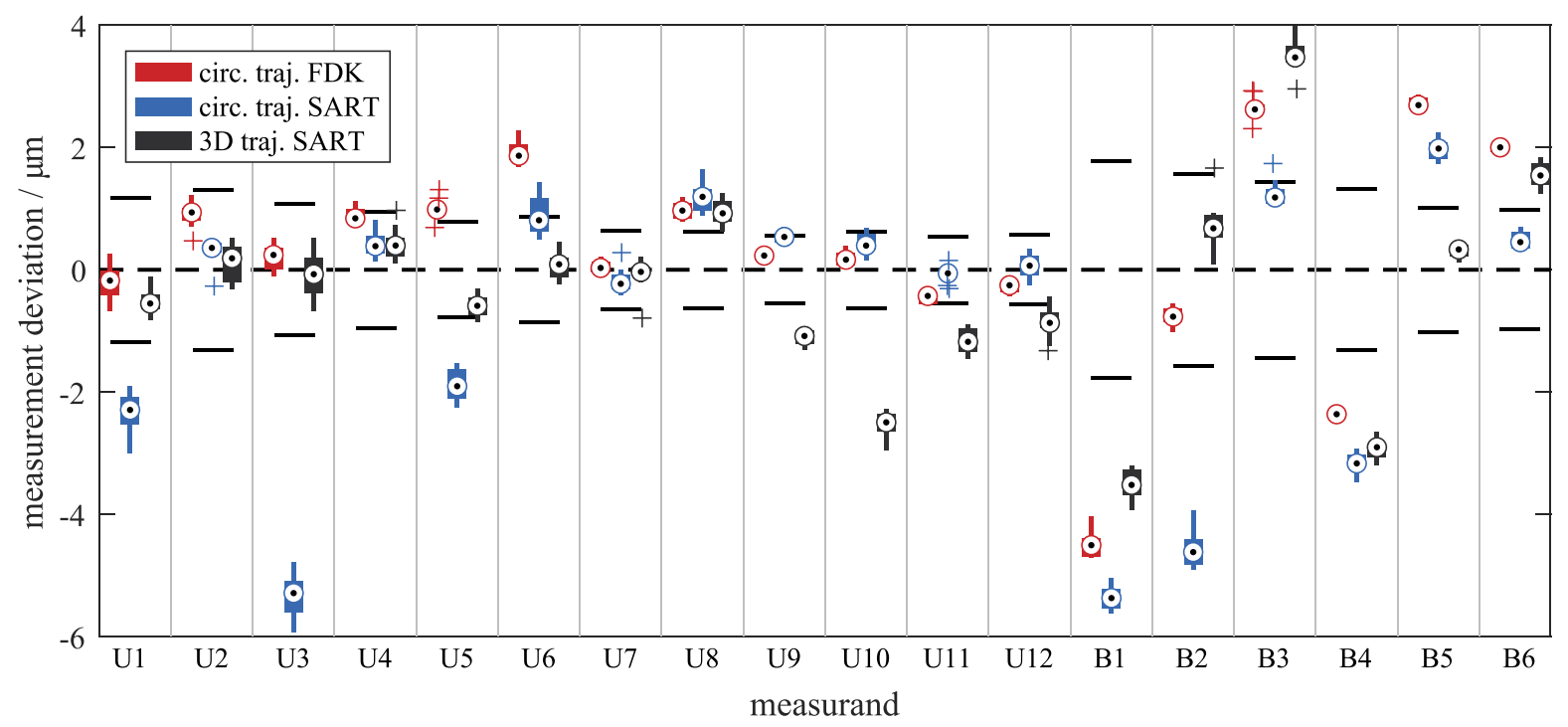

Figure 12. Dimensional measurement deviations for the unidirectional and bidirectional distances of the aluminum multi-feature specimen (see figure 3). The measurement uncertainties from the tactile reference measurement are indicated by the black horizontal lines.

further interest. The calibration procedure is an additional step that directly influences the overall machine time. However, if the same scan trajectory is applied several times, e.g. for geometrically identical parts of a production batch, this disadvantage diminishes.

Apart from the calibration, the additional movement of the hexapod axes cause increased scan times for single measurements. Furthermore, the iterative reconstruction technique, which is necessary to reconstruct the measurement volume for 3D scan trajectories, requires more computational time than the FDK reconstruction, which is typically used for circular scan trajectories. From table 2, it can be seen that for our investigations, the scan time for a $3 \mathrm{D}$ trajectory was $31 \%-$ $44 \%$ higher than the one for the respective circular trajectory. However, one has to note that scan times were not in focus when developing the program code for controlling the devices. For instance, the movement of the rotary table of the CT and the movement of the hexapod axes are performed sequentially, whereas they could also be performed in parallel.

Compared to the scan times, the differences in the reconstruction time are only relevant for the measurements with five iterations of the SART algorithm. However, even for these cases, the reconstruction time is lower than the scan time and would therefore not provoke any bottle neck in the measurement chain for industrial measurement series. Nevertheless, it has to be mentioned that the size of the reconstructed voxel data sets for our investigations was comparatively low (between $2.9 \times 10^{7}$ and $2.6 \times 10^{8}$ voxels). As the reconstruction time is proportional to the number of voxels, the reconstruction time can grow rapidly for larger data sets.

\section{Summary}

It was shown that complex 3D scan trajectories have been successfully applied for an industrial cone-beam CT scanner using a hexapod as additional manipulator system. The hexapod was used for adaptive tilting of the measurement object during the $360^{\circ}$ scan. This can be used to prevent unfavorable views for single projections and to avoid cone-beam artifacts. To enable proper reconstruction, off-line trajectory calibration based on a multi-sphere reference object was applied. For this purpose, existing methods from literature were modified to use available prior knowledge of the CT geometry.

Results for the demonstrator test fixture suggest that the greatest benefits from 3D scan trajectories can be expected for multi-material measurement tasks, where metal artifacts and truncation artifacts might not be avoidable for a circular scan trajectory. Although for more application-related parts such artifacts might not be completely avoidable using 3D scan trajectories, the strength might still be reducible with a proper choice of projective views.

Qualitative as well as quantitative results for the measured steel ball showed that cone-beam artifacts can be avoided for this part, which is not possible using a circular trajectory. This mainly had a positive effect regarding the determination of form deviations. Measurement deviations for the diameter of the ball could also be reduced, but this was mainly caused by the calibration procedure, which is able to compensate for geometrical misalignments of the assumed ideal geometry of the circular trajectory.

The evaluation of a variety of different length features for the multi-feature aluminum part allowed for figuring out the limits in terms of achievable accuracy. It was found that the use of the hexapod and the applied calibration procedure do not imply further significant systematic or random measurement deviations.

Results based on different calibration scans for the steel ball showed that the robustness of the calibration procedure could still be improved. It is assumed that this could be achieved by using a multi-sphere reference object with a higher number of spheres. Additionally, the spheres should be arranged in a 
way that the number of overlapping projected sphere contours is kept to a minimum for all possible poses that can be realized with the hexapod. Future work will therefore focus on comparing and improving different trajectory calibration methods.

It should be mentioned at this point that the hexapod and the presented calibration procedure could also be used for the fusion of single conventional cone-beam CT scans with different workpiece orientation, similar as proposed e.g. in [42-44].

The specific shapes of the 3D scan trajectories in this paper were chosen by an empirical approach. It is expected that the benefits from 3D trajectories could be further increased if more sophisticated methods based on mathematical optimization were applied. Thus, this will be an additional focus of our future research.

\section{Data availability statement}

The data that support the findings of this study are available upon reasonable request from the authors.

\section{Acknowledgments}

Author contributions according to CRediT taxonomy [45] are as follows: L B contributed conceptualization, data curation, formal analysis, investigation, methodology, project administration, software, visualization as well as writing-original draft. T H contributed funding acquisition, resources, supervision and writing - review and editing.

The authors thank the group of F Meli from the Federal Institute of Metrology (METAS), especially B Bircher, for providing the multi-sphere reference object and for helpful discussions. Gratitude is owed to M Krenkel and D Weiß (Carl Zeiss Industrielle Messtechnik $\mathrm{GmbH}$ ) for their help regarding the control of the hardware devices. Providing licenses and support for the reconstruction software by Siemens Healthcare GmbH (especially N Maaß and F Dennerlein) is gratefully acknowledged, as well as support by A Torba and J Schinn regarding the construction/mounting of measurement objects and fixtures and $\mathrm{P}$ Kaller regarding the reference measurements. The acquisition of the CT system Metrotom 1500 was financially supported by the German Research Foundation DFG through Grant No. 324672600.

\section{ORCID iDs}

Lorenz Butzhammer (D) https://orcid.org/0000-0002-55374849

Tino Hausotte (D) https://orcid.org/0000-0002-2923-3217

\section{References}

[1] Ametova E, Probst G and Dewulf W 2018 X-ray computed tomography devices and their components Industrial X-Ray Computed Tomography ed S Carmignato, W Dewulf and R Leach (Cham: Springer International Publishing) pp 69-98
[2] De Chiffre L, Carmignato S, Kruth J P, Schmitt R and Weckenmann A 2014 Industrial applications of computed tomography CIRP Ann. Manuf. Technol. 63 655-77

[3] Villarraga-Gómez H, Herazo E L and Smith S T 2019 X-ray computed tomography: from medical imaging to dimensional metrology Precis. Eng. 60 544-69

[4] Tuy H 1983 An inversion formula for cone-beam reconstruction SIAM J. Appl. Math. 43 546-52

[5] Buzug T M 2008 Computed Tomography: From Photon Statistics to Modern Cone-Beam CT (Berlin: Springer)

[6] Beister M, Kolditz D and Kalender W A 2012 Iterative reconstruction methods in x-ray CT Phys. Medica 28 94-108

[7] Capostagno S, Stayman J W, Jacobson M W, Ehtiati T, Weiss C R and Siewerdsen J H 2019 Task-driven source-detector trajectories in cone-beam computed tomography: II. Application to neuroradiology J. Med. Imaging 6025004

[8] Hiller J, Landstorfer P, Marx P and Herbst M 2020 Evaluation of the impact of faulty scanning trajectories in robot-based x-ray computed tomography Meas. Sci. Technol. 32015401

[9] Klein P and Herold F 2016 Automatic object position recognition: increasing the position-accuracy in robot-CT 6th Conf. Industrial Computed Tomography (iCT 2016) (Wels, Austria)

[10] Banjak H, Costin M, Vienne C, Guillamet R and Kaftandjian V 2017 Iterative CT reconstruction on limited angle trajectories applied to robotic inspection AIP Conf. Proc. 1806020009

[11] Vienne C and Costin M 2018 Adapted acquisition trajectory and iterative reconstruction for few-views CT inspection 8 th Conf. Industrial Computed Tomography (iCT 2018) (Wels, Austria)

[12] Holub W, Brunner F and Schön T 2019 RoboCT application for in-situ inspection of join technologies of large scale objects Int. Symp. Digital Industrial Radiology and Computed Tomography—DIR2019

[13] Krumm M, Sauerwein C, Hämmerle V, Heile S, Schön T, Jung A and Sindel M 2018 Rapid robotic x-ray computed tomography of large assemblies in automotive production 8th Conf. Industrial Computed Tomography (iCT 2018) (Wels, Austria)

[14] Kang R, Probst G M, Slaets P and Dewulf W 2020 Investigation of the impact of various robot properties on a twin robot-CT system Nondestruct. Test. Eval. $35276-86$

[15] VDI/VDE 2630 Part 132011 Computed tomography in dimensional measurement-guideline for the application of DIN EN ISO 10360 for coordinate measuring machines with CT sensors

[16] Fischer A, Lasser T, Schrapp M, Stephan J and Noël P B 2016 Object specific trajectory optimization for industrial x-ray computed tomography Sci. Rep. 619135

[17] Herl G, Hiller J and Maier A 2020 Scanning trajectory optimisation using a quantitative Tuy-based local quality estimation for robot-based x-ray computed tomography Nondestruct. Test. Eval. 35 287-303

[18] Schabunow A 2018 Einstellung von Aufnahmeparametern mittels projektionsbasierter Qualitätskenngrößen in der industriellen Röntgen-Computertomographie PhD Thesis Karlsruher Institut für Technologie (KIT)

[19] Heinzl C, Kastner J, Amirkhamov A, Gröller E and Gusenbauer C 2012 Optimal specimen placement in cone beam x-ray computed tomography NDT\&E Int. 50 42-9

[20] Butzhammer L, Müller A M and Hausotte T 2020 Comparison of geometrically derived quality criteria regarding optimal workpiece orientation for computed tomography measurements 10th Conf. Industrial Computed Tomography (iCT 2020) (Wels, Austria) 
[21] Xue L and Suzuki H 2017 Evaluation of scanning parameters based on image entropy for dimensional computed tomography metrology J. Manuf. Sci. Eng. 139071001

[22] Wood C E, O'Brien N, Denysov A and Blumensath T 2019 Computed laminography of CFRP using an x-ray cone-beam and robotic sample manipulator systems IEEE Trans. Nucl. Sci. 66 655-63

[23] Weiß D, Lonardoni R, Deffner A and Kuhn C 2012 Geometric image distortion in flat-panel $\mathrm{x}$-ray detectors and its influence on the accuracy of CT-based dimensional measurements 4th Conf. Industrial Computed Tomography (iCT 2012)

[24] Butzhammer L and Hausotte T 2019 Effect of iterative sparse-view CT reconstruction with task-specific projection angles on dimensional measurements 9th Conf. Industrial Computed Tomography (iCT 2019)

[25] DIN 5401:2002-08 2002 Rolling Bearings_-Balls for Rolling Bearings and General Industrial Use (Deutsches Institut für Normung)

[26] VDI/VDE 2617 Part 72008 Accuracy of coordinate measuring machines-parameters and their checking—estimation of measurement uncertainty of coordinate measuring machines by means of simulation

[27] Feldkamp L A, Davis L C and Kress J W 1984 Practical cone-beam algorithm J. Opt. Soc. Am. A 1 612-9

[28] Siemens Healthcare GmbH 2019 CERA API User Manual, English, Version 6

[29] Andersen A H and Kak A C 1984 Simultaneous algebraic reconstruction technique (SART): a superior implementation of the art algorithm Ultrason. Imaging 6 81-94

[30] Blumensath T, O'Brien N and Wood C E 2018 Calibration of robotic manipulator systems for cone-beam tomography imaging IEEE Trans. Nucl. Sci. 65 1384-93

[31] Hartley R and Zisserman A 2003 Multiple View Geometry in Computer Vision 2nd edn (Cambridge: Cambridge University Press)

[32] Li X, Zhang D and Liu B 2010 A generic geometric calibration method for tomographic imaging systems with flat-panel detectors-a detailed implementation guide $\mathrm{Med}$. Phys. 37 3844-54
[33] Küng A, Meli F and Thalmann R 2007 Ultraprecision micro-CMM using a low force 3D touch probe Meas. Sci. Technol. 18 319-27

[34] Bircher B A, Meli F, Küng A and Thalmann R 2019 CT geometry determination using individual radiographs of calibrated multi-sphere standards 9th Conf. Industrial Computed Tomography (iCT 2019) (Padova, Italy)

[35] Canny J 1986 A computational approach to edge detection IEEE Trans. Pattern Anal. Mach. Intell. PAMI-8 679-98

[36] Deng L, Xi X, Li L, Han Y and Yan B 2015 A method to determine the detector locations of the cone-beam projection of the balls' centers Phys. Med. Biol. 60 9295-311

[37] Müller P, Hiller J, Dai Y, Andreasen J L, Hansen H N and De Chiffre L 2015 Quantitative analysis of scaling error compensation methods in dimensional x-ray computed tomography CIRP J. Manuf. Sci. Technol. 10 68-76

[38] Dodge Y 2008 Coefficient of determination The Concise Encyclopedia of Statistics (New York: Springer) pp 88-91

[39] Maaß C, Knaup M and Kachelrieß M 2011 New approaches to region of interest computed tomography Med. Phys. 38 2868-78

[40] Bartolac S, Clackdoyle R, Noo F, Siewerdsen J, Moseley D and Jaffray D 2009 A local shift-variant Fourier model and experimental validation of circular cone-beam computed tomography artifacts Med. Phys. 36 500-12

[41] Ferrucci M, Dewulf W and Dönmez A 2021 Measurement of sample stage error motions in cone-beam $\mathrm{x}$-ray computed tomography instruments by minimization of reprojection errors Precis. Eng. 67 48-57

[42] Herl G, Hiller J, Kasperl S and Maier A 2020 Reduction of metal artifacts for industrial computed tomography using multipositional data fusion Tech. Mess. tm 87 101-10

[43] Kostenko A, Andriiashen V and Batenburg K J 2018 Registration-based multi-orientation tomography $\mathrm{Opt}$. Express 26 28982-95

[44] Müller A M and Hausotte T 2018 Data fusion of surface data sets of $\mathrm{x}$-ray computed tomography measurements using locally determined surface quality values J. Sens. Sens. Syst. 7 551-7

[45] CASRAI CRediT (www.casrai.org/credit.html) (accessed 11 June 2021) 